\title{
A New System Splitting Scheme Based on the Unified Stability Control Framework
}

\author{
Ming Jin, Tarlochan S. Sidhu, Fellow, IEEE, and Kai Sun
}

\begin{abstract}
In this paper, a new system splitting scheme based on the unified stability control framework is proposed. Compared with the conventional system splitting scheme, the new method is much more effective under complicated oscillation scenarios. Under the unified stability control framework, different emergency control measures can be evaluated and compared with each other. The system splitting strategy will be applied to the system only when the analysis shows that the splitting is necessary or reasonable. Related algorithms are described in detail in this paper. The advantages and effectiveness of the new scheme can be justified through the testing cases.
\end{abstract}

Index Terms-Complementary-cluster center-of-inertia relative motion (CCCOI-RM) transform, controlling group, emergency control, system splitting, transient stability margin.

\section{INTRODUCTION}

$\mathbf{T}$ RADITIONALLY, if the power system enters the emergency mode due to some disturbances, emergency controls will be applied to drive the system back to the alert mode. If the emergency controls fail to bring the system parameters back within their constraints, protective devices will act to prevent the power system components operating under unacceptable conditions from undergoing irrevocable damages [1]. This may lead to system islanding, which often produces unstable or generation-load unbalanced islands. The system splitting (also called controlled system islanding) discussed in this paper is quite different. It means that the dispatching center splits the power network into several maintainable islands actively by opening selected lines when no appropriate control measures are reasonable or the control cost is too high. Here, "maintainable" means that some necessary conditions, such as transient stability, generation-load balance, and transmission capacity constraints, should be satisfied in each island after splitting. From this point of view, the system splitting should also be thought as one emergency control method [4].

So far, most of the splitting schemes used in actual systems are based on the concept of coherency. However, when the oscillation mode of the power system is very complicated, the coherent splitting scheme may encounter some difficulties. First, theoretical analysis and computer simulation shows that simply splitting the system from the coherent surface cannot always

Manuscript received December 13, 2005; revised May 25, 2006. Paper no. TPWRS-00825-2005

The authors are with the Department of Electrical and Computer Engineering, The University of Western Ontario, London, ON N6A 5B9, Canada (e-mail: mjin4@uwo.ca).

Digital Object Identifier 10.1109/TPWRS.2006.887891 guarantee the stability in each island directly [8], [9]. Due to the difficulty of defining quantitative stability margin index based on the coherent group information, it is very difficult to design and assess the succeeding control measures in advance. Second, the identification of the coherent group itself under complicated oscillation scenarios is also challenging, especially when system oscillates in multicluster mode with high incoherency within one or more cluster(s).

Furthermore, in the history of power system study, the system splitting is studied separately from the emergency controls because of the different system characteristics after the controls are applied, e.g., the system splitting will cause significant changes to the network structure, and the integrity of the power grid will be damaged. Without a unified decision-making mechanism, it is very difficult to assess and compare the control effects and costs between the system splitting and other emergency control measures. For example, it is difficult to answer the following basic question: when is the system splitting necessary?

In this paper, a new system splitting scheme based on the unified stability control framework is proposed. Compared with the conventional system splitting scheme, the new method is much more effective under complicated oscillation scenarios. Under the unified stability control framework, different emergency control measures can be evaluated and compared with each other. The system splitting strategy will be applied to the system only when the analysis shows that the splitting is necessary or reasonable. Related algorithms are described in detail in this paper. The advantages and effectiveness of the new scheme can be justified through the testing cases.

\section{CONTROLLING GROUP AND EMERGENCY CONTROL}

The new system splitting scheme proposed in this paper is based on the identification of the controlling group of generators. The controlling group is the leading group for the controlling mode [4]. For each analytical scenario, the swing trajectories of a system with $n$ generators can be divided into complementary groups in $n-1$ different ways from the angle space of any two adjacent trajectories. Each pair of complementary group can be represented by an equivalent two-machine mapping system, further, one-machine infinite-bus (OMIB) mapping system through the complementary-cluster center-of-inertia relative motion (CCCOI-RM) transform. The OMIB mapping trajectory with the smallest stability margin is corresponding to the controlling mode. The algorithm used to determine the controlling group can be described as follows [1], [2], [4].

1) At each time step of the post-fault simulation, rank the trajectories according to the rotor angles. 
2) For each angle space, divide the trajectories into two groups, $S$ group and $A$ group, according to their location above or below the angle space.

3) In each group, aggregate the trajectories via the CCCOI transform

$$
\begin{aligned}
& \delta_{S}(t)=\sum_{i \in S} M_{i} \delta_{i}(t) / \sum_{i \in S} M_{i} \\
& \delta_{A}(t)=\sum_{j \in A} M_{j} \delta_{j}(t) / \sum_{j \in A} M_{j}
\end{aligned}
$$

where $\delta$ is the rotor angle, and $M$ is the moment of inertia.

4) Apply the RM transform to the complementary group to acquire a time variant equivalent OMIB trajectory

$$
\delta(t)=\delta_{S}(t)-\delta_{A}(t)
$$

The corresponding OMIB rotor speed is expressed as

$$
\omega(t)=\omega_{S}(t)-\omega_{A}(t)
$$

where

$$
\begin{aligned}
& \omega_{S}(t)=\sum_{i \in S} M_{i} \omega_{i}(t) / \sum_{i \in S} M_{i} \\
& \omega_{A}(t)=\sum_{j \in A} M_{j} \omega_{j}(t) / \sum_{j \in A} M_{j} .
\end{aligned}
$$

The equivalent OMIB mechanical power is defined as

$P_{m}(t)=\left(M_{A} \sum_{i \in S} P_{m i}-M_{S} \sum_{j \in A} P_{m j}\right) /\left(M_{S}+M_{A}\right)$

and the equivalent OMIB electrical power as

$$
P_{e}(t)=\left(M_{A} \sum_{i \in S} P_{e i}-M_{S} \sum_{j \in A} P_{e j}\right) /\left(M_{S}+M_{A}\right)
$$

where

$$
M_{S}=\sum_{i \in S} M_{i} ; \quad M_{A}=\sum_{j \in A} M_{j} .
$$

The equivalent OMIB inertia coefficient will be

$$
M=\frac{M_{S} M_{A}}{M_{S}+M_{A}} .
$$

5) Analyze the equivalent OMIB trajectory on the $P-\delta$ plane. If a dynamic saddle point (DSP) is found on the trajectory, the corresponding trajectory can be judged as an unstable one. The stability margin can be calculated as

$$
\eta=A_{\text {dec }}-A_{\text {inc }}
$$

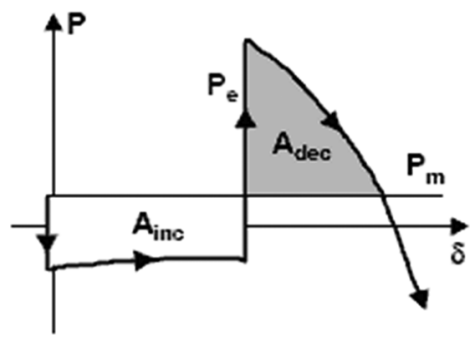

Fig. 1. Definition of the transient stability margin index.

where $A_{d e c}, A_{i n c}$ are decelerating and accelerating area on the OMIB $P-\delta$ plane, respectively, as shown in Fig. 1 [11].

In practice, a more convenient way is often used to calculate the stability margin

$$
\eta=-E_{K \cdot D S P}=-\frac{1}{2} M \omega_{D S P}^{2}
$$

where $E_{K . D S P}$ and $\omega_{D S P}$ are the kinetic energy and rotor speed when the equivalent OMIB trajectory reaches the DSP.

If a return point (RP) is found on the trajectory, the corresponding trajectory can be judged as stable in this particular swing. The stability margin can be calculated as

$$
\eta=\int_{\delta_{R P}}^{\delta_{D S P}}\left(P_{e}-P_{m}\right) d \delta .
$$

The triangle (TRI) or weighted least square (WLS) approximation [1] based on the sensitivity calculation may be used to estimate the imaginary DSP.

6) Apply the algorithm to each angle space, the equivalent OMIB trajectory with the smallest stability margin will be the controlling trajectory, and the corresponding leading group is the controlling group.

For the emergency control, the importance of the controlling group identification comes from the following facts: first, the controlling group provides the identification of generators that should be responsible for the system out of step condition [4]. It will indicate the objects that the controls should be applied to; second, the corresponding stability margin index can be used to assess the degree of system stability and the control effects. For a given fault scenario, the controlling mode, further, the controlling group, is related to a fixed point in the parameter space and can be identified through the corresponding swing trajectories directly. The changing of the system parameter will lead to the changing of controlling group accordingly. If the emergency control problem is observed in the parameter space, no matter which kind of measures will be applied, all of them are trying to change the system oscillation mode from unstable to stable by changing one or more system parameter(s). In general, the nature of the emergency control decision-making process will be an iterative one, and the sensitivity analysis is important in the process. 


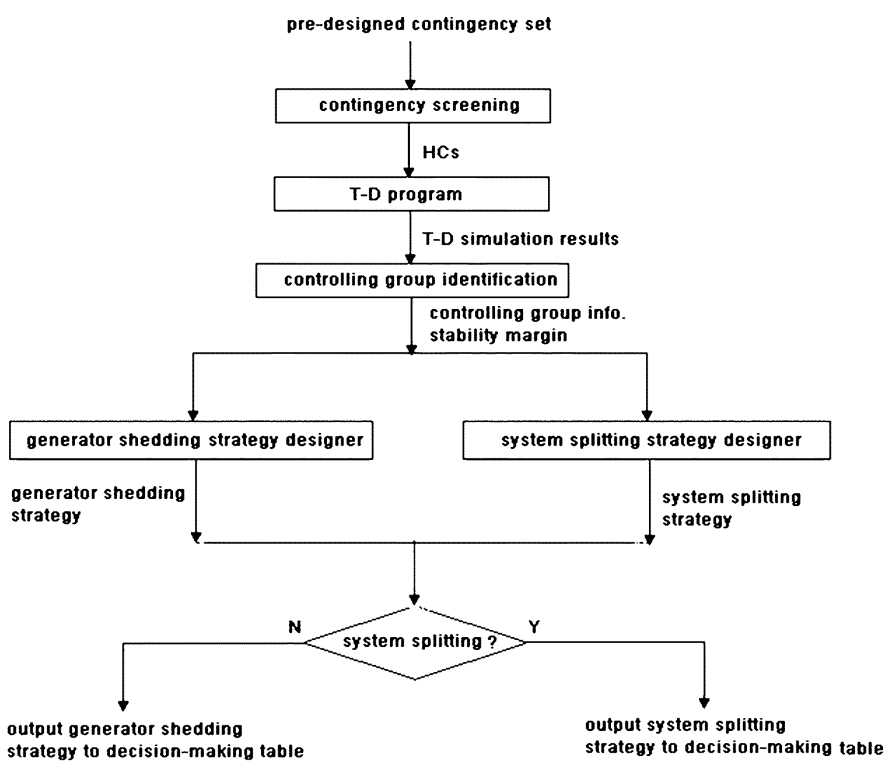

Fig. 2. Proposed stability control framework.

\section{UNIFIED EMERGENCY CONTROL FRAMEWORK}

It is well known that the emergency controls in large-scale power systems can be implemented in three different ways: offline analysis, real-time matching; online analysis, real-time matching; and real-time analysis, real-time control [4]. The emergency control scheme proposed in this paper is based on the online analysis, real-time matching mode. It means that the pre-analysis will just focus on the current operational scenario and should be completed before the occurrence of the disturbance; after a disturbance occurs in the power system, an appropriate control measure will be chosen from the decision-making table and be applied to the system. The details of the proposed stability control framework can be shown in Fig. 2.

In the online pre-analysis stage, system swing trajectories will be obtained via the time-domain transient stability analysis program (T-D program). For all the possible contingencies that may occur in power systems, only a small portion of them may drive the system into the unstable state. Also, just for these unstable cases is the corresponding emergency control needed. In order to ensure the speed of the whole analytical process and decrease the computational burden, a fast contingency screening module will be used before the detailed analysis. The contingency screening module scans the pre-designed contingency set with a similar simulation mechanism that will be used in detailed analysis but a simplified system model. Then, the contingencies are ranked based on the critical clearing time (CCT) or stability margin criterion. Only the so-called "harmful contingencies (HCs)" [1] will be submitted to the analytical mechanism, and the detailed dynamic process during the fault-on and post-fault stage, i.e., the swing trajectories, will be simulated.

After the swing trajectories are obtained, they will be sent to the controlling group identification module. The module tests the possible decomposition patterns to find the controlling mode, therefore, the controlling group, and calculate the corresponding stability margin index based on the algorithm de- scribed in Section II. Then, the related information will be used to design the system splitting strategy and other user-selected emergency control scheme(s), such as generator shedding, fast valving, load shedding, etc. In this paper, generator shedding is used as an example to show the details of the algorithm.

Then, the comparison between the system splitting and userselected emergency control scheme(s) will be made based on the user-defined criteria, such as the total quantity of load losses, the deviation of system frequency during the transient process, etc. Finally, the chosen strategy will be added to the decisionmaking table.

\section{Design of Control StRategies}

As discussed above, the generator shedding is chosen as an example to show the capability of the new proposed unified emergency control framework. The generator shedding has been studied extensively, and detailed discussion can be found in [1]-[4]. The algorithm for generator shedding used in this paper can be described as follows.

1) Calculate the sensitivity coefficient $S_{P m}^{\eta}$ via the numerical perturbation method

$$
S_{P m}^{\eta}=\frac{\eta\left(P_{m 0}+\Delta P_{m}\right)-\eta\left(P_{m 0}\right)}{\Delta P_{m}} .
$$

Here, the parameter $P_{m}$ is the mechanical power of the controlling OMIB equivalent system.

2) Estimate the mechanical input power that should be removed from the system based on the sensitivity coefficient described in step 1. If the system loses its synchronism during the forward swing, the power should be removed from the controlling group; otherwise, it should be removed from the remaining group.

3) Choose the candidate removing generators in the appropriate group following one of the rules described below:

- most advanced unit;

- unit with the largest $P_{m} / M$ radio.

Repeat step 3 until sufficient injection power has been removed.

4) Execute the T-D simulation, modify the CCCOI-RM mapping, and calculate the modified stability margin.

5) If necessary, repeat steps 1-4 until the stability margin becomes appropriate.

As the focus of this paper, the system splitting is studied in detail. It is well known that the most important and critical problem for the system splitting is how to decide the splitting surface. From the view of energy, if the system loses its synchronism during the forward swing, some injection energy should be removed from the leading group; if the system loses its synchronism during the backward swing, some injection energy should be removed from the remaining group. So the simplest way to determine the system splitting strategy is to split the power network just from the controlling group surface. There are two possible ways to search the splitting surface: analytical and heuristic method.

The ordered binary decision diagram (OBDD)-based method described in [8] is a typical analytical algorithm. It searches the possible splitting strategies through the following three phases. 
Phase-1: Construct a graph-theory model for the large power network, and reduce it based on the graph theory and power system characteristics.

Phase-2: Search for all possible strategies that can be used to separate controlling and remaining group of generators and create generation-load balanced islands.

Phase-3: Check the other conditions, e.g., transmission capacity constraints, and give out candidate splitting strategies.

The analytical method solves the system splitting problem just from the mathematical view. The existing knowledge and experience for a particular system can not be utilized sufficiently. Furthermore, the splitting strategy proposed by the analytical method often does not fit the custom of power system operation, e.g., in practice, the system splitting will only occur from some critical tie lines but not anywhere in the network. Compared with the analytical one, the heuristic method seems more efficient and suitable to this problem. With the heuristic method, the whole network will be divided into several areas according to the system knowledge and operational experience, e.g., power source distribution, generator capacity, load characteristics, etc. The planning islands will be the combination of these areas. In this scheme, the system splitting can only occur from the interconnected lines between these areas. Although the heuristic method is not the key advantage of the new splitting scheme, its merits include lower online calculation burden for splitting surface searching, less splitting and communication equipments requirement, consistent with the existing system operation practices, easy to be accepted by field engineers, etc. The splitting surface determination in this paper is realized following the heuristic way.

The algorithm for the system splitting strategy designer can be described as follows.

1) Search for the splitting strategy by the analytical or heuristic method described above.

2) Execute the T-D simulation, modify the CCCOI-RM mapping in each island, and calculate the corresponding stability margins.

3) If the stability margin becomes appropriate in each island, output the splitting strategy.

4) Otherwise, further control measures will be proposed via the additional control designer.

Here, a very important point is that no matter which kind of splitting scheme will be applied, the stability on each island after the splitting cannot be guaranteed directly. The reason is that the system splitting operation itself can be thought of as a large disturbance to the system, and consequence of this disturbance cannot be predicted from the previous simulation trajectories directly, because the extension of any time sequence data is only suitable to the process in which the parameters change continuously [4]. So, the proposed splitting strategy will be assessed via the T-D program again. If the assessment shows that the stability cannot be reached in one or more island(s), further control measures will be designed via the additional control designer module.

The comparison between the two splitting methods (coherency based and controlling group based) is as follows. According to single machine equivalent (SIME) or integrated extended equal area criterion (IEEAC) theory, if the system oscillates in two clusters and strict coherency exists in each cluster, the two splitting

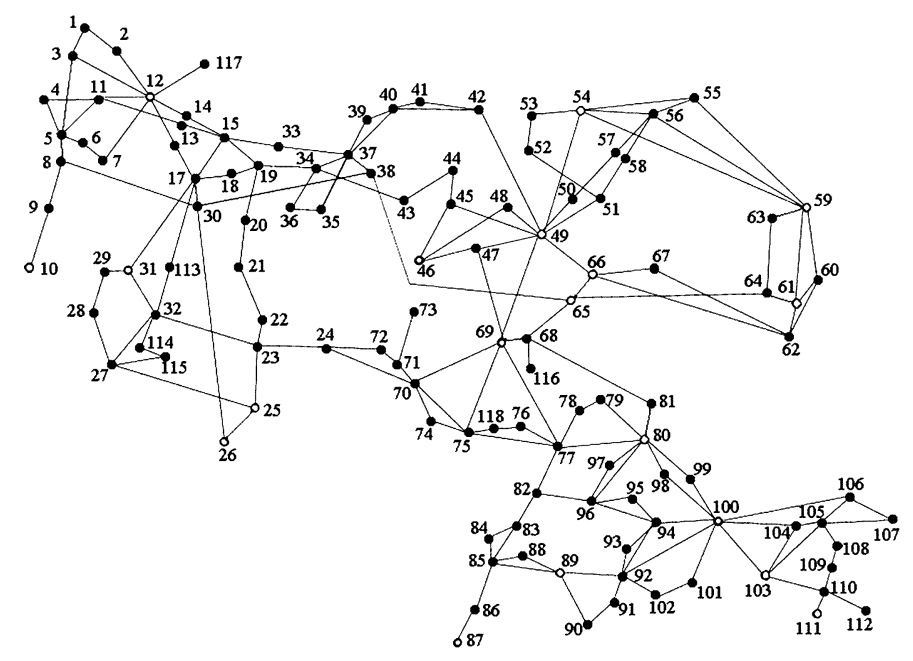

Fig. 3. IEEE 118-bus system.

methods are exactly equivalent. The advantage of the new proposed splitting scheme will be shown when system oscillates in complicated scenarios. In theory, the controlling group-based splitting scheme tries to split the system into two islands irrespective of the system complexity and the number of clusters in which the system oscillates. However, in some cases, the system may have to be split into more than two islands due to the constraints of system topology and operation feasibility. If the stability in one or more island(s) is not satisfied, additional controls, such as generator shedding, will be applied to avoid the further spitting. By trying to separate the system into fewer islands, the integrity of the network will be improved.

\section{Test Cases}

In this paper, the IEEE 118-bus (19 generators) system is chosen as the research platform. The system structure is shown in Fig. 3.

The purpose of testing is to show the advantages and effectiveness of the proposed scheme. Different control strategies, such as generator shedding, conventional coherent splitting, and the controlling group-based splitting, will be compared under the unified stability control framework.

Case 1: A three-phase-to-ground fault occurred on line $26-30$ and is very close to bus 30 . The fault is cleared by protective relays at $0.3 \mathrm{~s}$ after the fault occurs.

Here, the time settings of the protective relays are carefully chosen to show the desired oscillation mode, although, in practice, they may be shorter than $0.3 \mathrm{~s}$

The swing trajectories obtained from the T-D program are shown in Fig. 4.

The generator shedding strategy designer proposes the following strategy:

Shedding generator at bus 25 ;

Shedding generator at bus 26 ;

Shedding generator at bus 10 .

Fig. 5 shows the simulation results after the above generator shedding strategy is applied to system. The generators are shed at $0.4 \mathrm{~s}$ after the fault occurs. 




Fig. 4. Swing trajectories for Case 1.

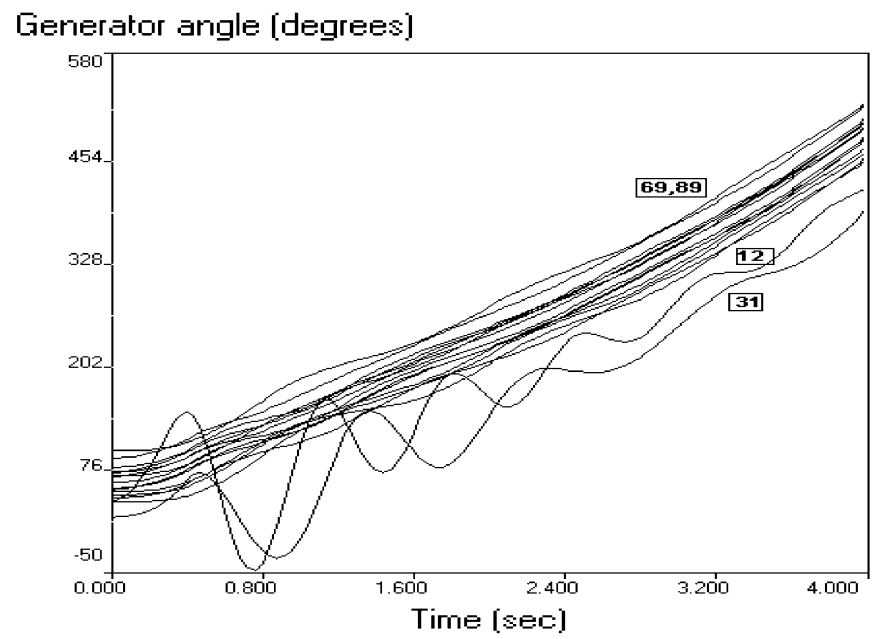

Fig. 5. Swing trajectories for Case 1 after the generator shedding.

Just from the view of stability, the generator shedding scheme described above is acceptable. However, the scheme will bring up to 984-MW load losses in the system. At the same time, the system splitting strategy designer proposes the following splitting scheme:

Remove line between bus 17 and 30;

Remove line between bus 8 and 30;

Remove line between bus 15 and 33;

Remove line between bus 24 and 70;

Remove line between bus 24 and 72;

Remove line between bus 19 and 34 .

Figs. 6 and 7 show the simulation results after the above system splitting strategy is applied to the system. The splitting is executed at $0.4 \mathrm{~s}$ after the fault occurs.

The topology of the power network after the system splitting is shown in Fig. 8. The whole system is split into two islands. The mismatching powers in the two islands are 63.00 and $-63.00 \mathrm{MW}$, respectively (the losses of the power network have been handled in advance). So the scheme only brings no more than 63-MW load losses in the system. Just from the view of the quantity of load losses, the splitting scheme is more efficient. However, the splitting scheme will bring nearly $3-\mathrm{Hz}$ frequency deviation on one island. In this case, the final choice will be

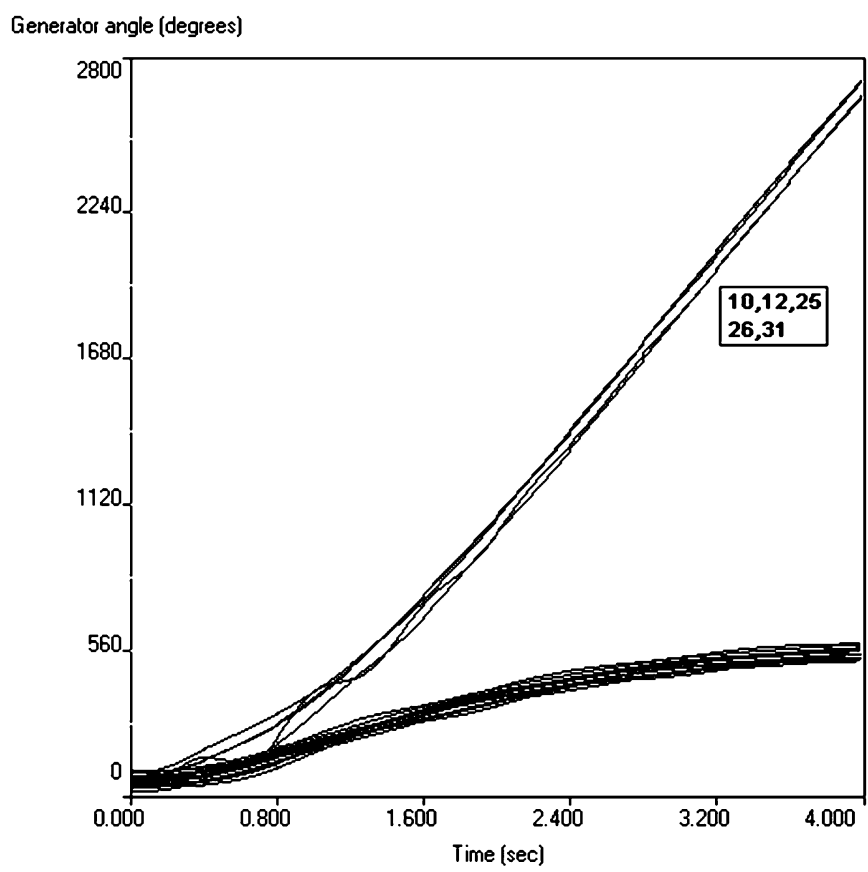

Fig. 6. Swing trajectories for Case 1 after controlling group-based splitting.

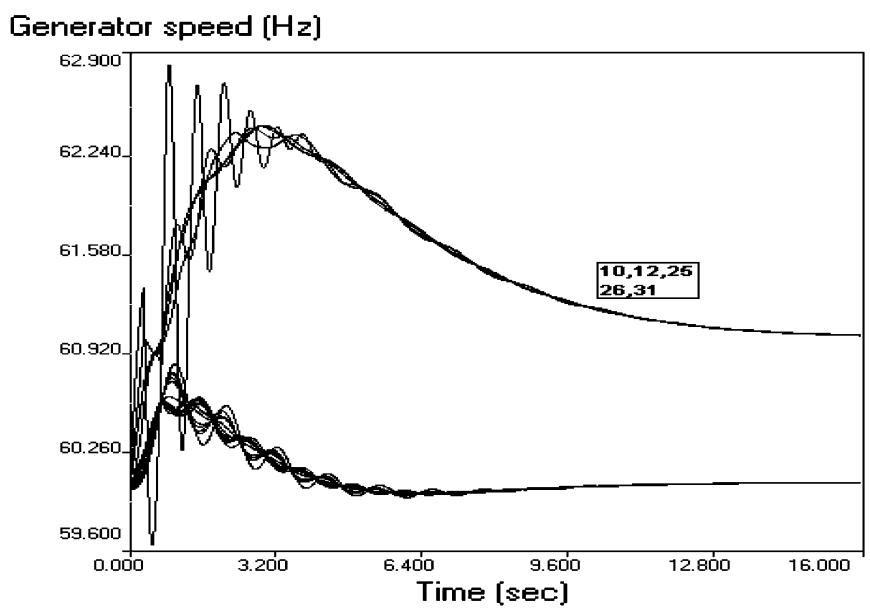

Fig. 7. System frequency for Case 1 after controlling group-based splitting.

made based on the user-defined criterion, or other control measures, such as fast valving, can be applied first to decrease the frequency deviation.

For comparison, the conventional splitting strategy based on the coherent group proposes the following splitting scheme:

Remove line between bus 17 and 31;

Remove line between bus 17 and 113;

Remove line between bus 17 and 18;

Remove line between bus 15 and 19;

Remove line between bus 17 and 30;

Remove line between bus 8 and 30;

Remove line between bus 15 and 33;

Remove line between bus 24 and 70;

Remove line between bus 24 and 72;

Remove line between bus 19 and 34 . 




Fig. 8. Network topology for Case 1 after controlling group-based splitting.

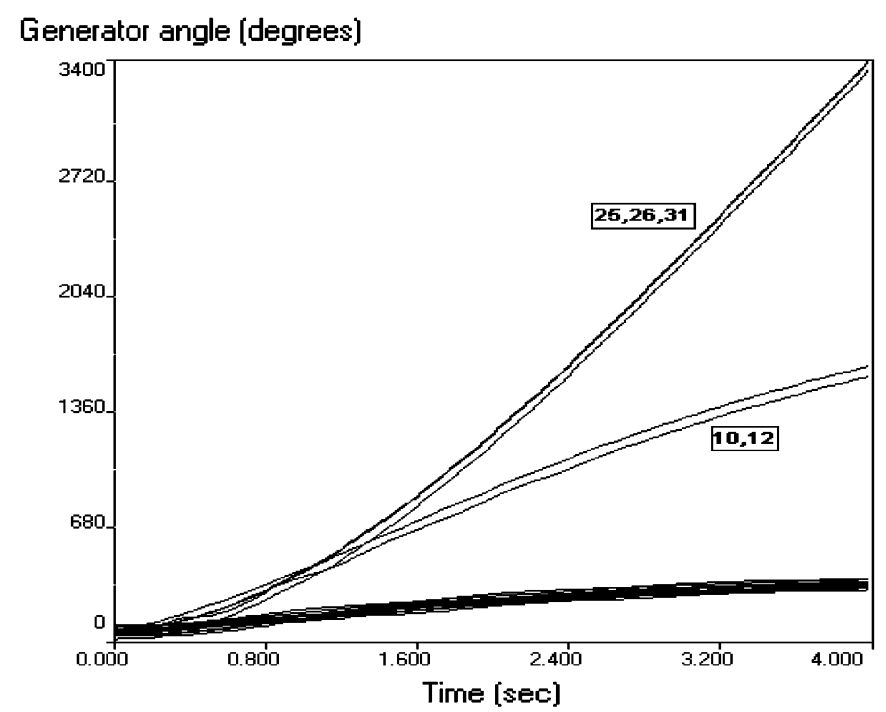

Fig. 9. Swing trajectories for Case 1 after coherent group-based splitting.

Fig. 9 shows the simulation results after the above system splitting strategy is applied to system. The splitting is executed at $0.4 \mathrm{~s}$ after the fault occurs. The topology of the power network after the system splitting is shown in Fig. 10.

The whole system is split into three islands. The mismatching powers in the three islands are $-41.70,104.70$, and -63.00 MW, respectively. So the scheme may bring 104.7-MW load losses in the system. Compared with the generator shedding scheme, the scheme is much better. However, it is obviously worse than the controlling group-based splitting scheme.

Case 2: A three-phase-to-ground fault occurred on the line $23-25$ and is very close to bus 25 . The fault is cleared by protective relays at $0.3 \mathrm{~s}$ after the fault occurs.

Here, the time settings of the protective relays are carefully chosen to show the desired oscillation mode, although, in practice, they may be shorter than $0.3 \mathrm{~s}$

The swing trajectories obtained from the T-D program are shown in Fig. 11.

The generator shedding strategy designer proposes the following strategy:

Shedding generator at bus 25 ;

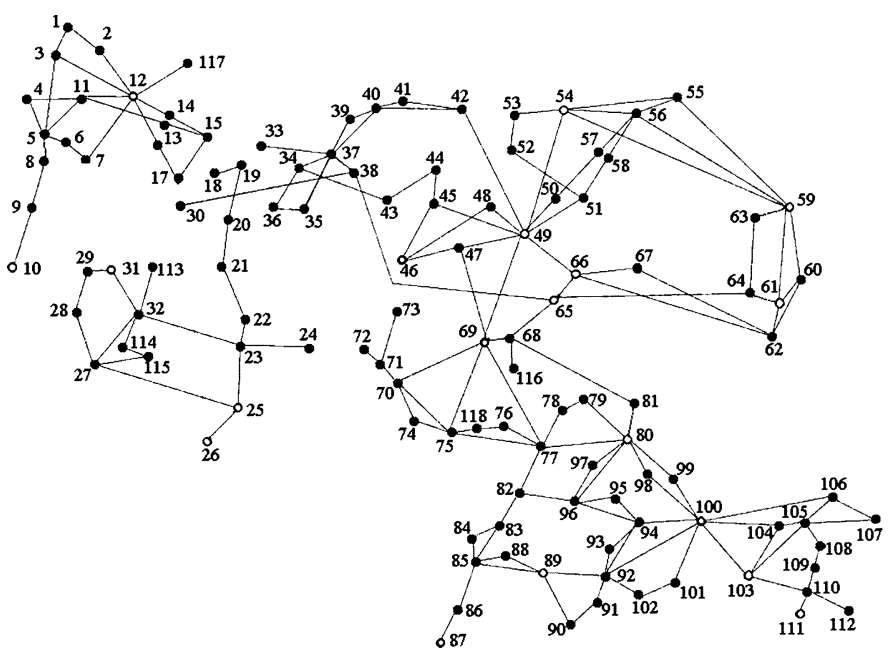

Fig. 10. Network topology for Case 1 after coherent group-based splitting.

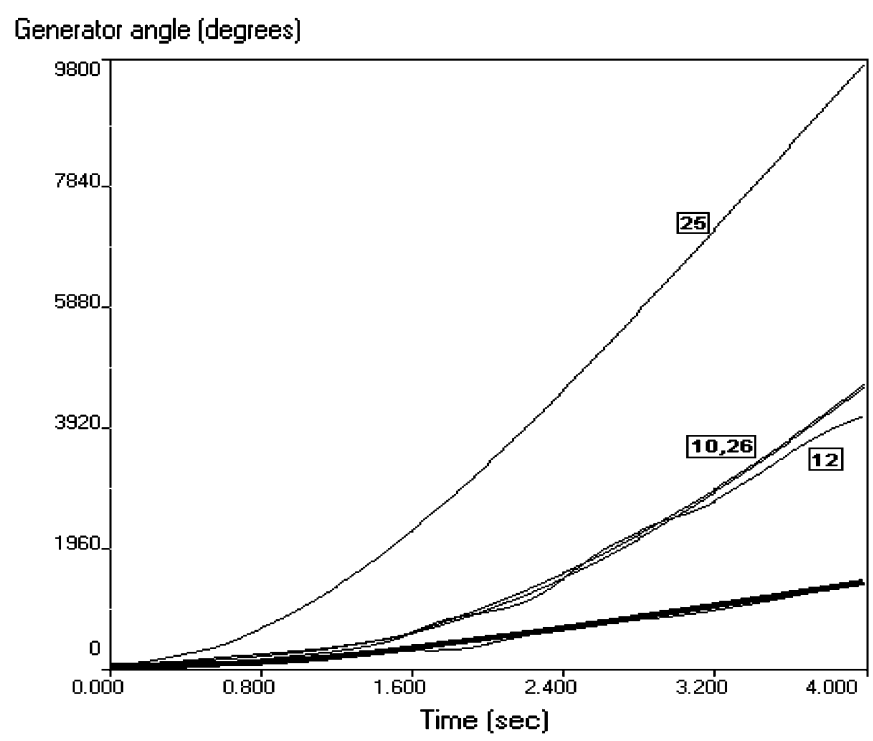

Fig. 11. Swing trajectories for Case 2 .

Figs. 12 and 13 show the simulation results after the above generator shedding strategy is applied to system. The generators are shed at $0.4 \mathrm{~s}$ after the fault occurs.

The generator shedding scheme described above will bring up to 220-MW load losses in the system. At the same time, the system splitting strategy designer and additional control designer propose the following splitting scheme:

Remove line between bus 25 and 26;

Remove line between bus 17 and 31;

Remove line between bus 17 and 113;

Remove line between bus 23 and 32;

Shedding generator at bus 31 .

Figs. 14 and 15 show the simulation results after the above system splitting strategy is applied to the system. The splitting is executed at $0.4 \mathrm{~s}$ after the fault occurs.

The whole system will be split into two islands. The mismatching powers in the two islands are -31.00 and $31.00 \mathrm{MW}$, respectively. So the scheme only brings no more than 36-MW 


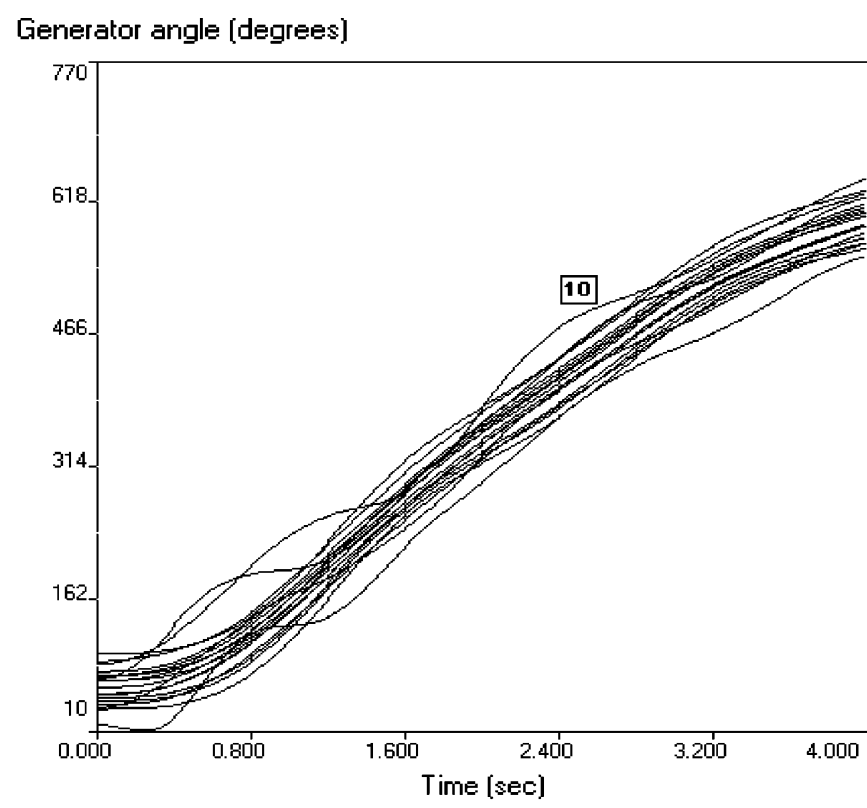

Fig. 12. Swing trajectories for Case 2 after the generator shedding.

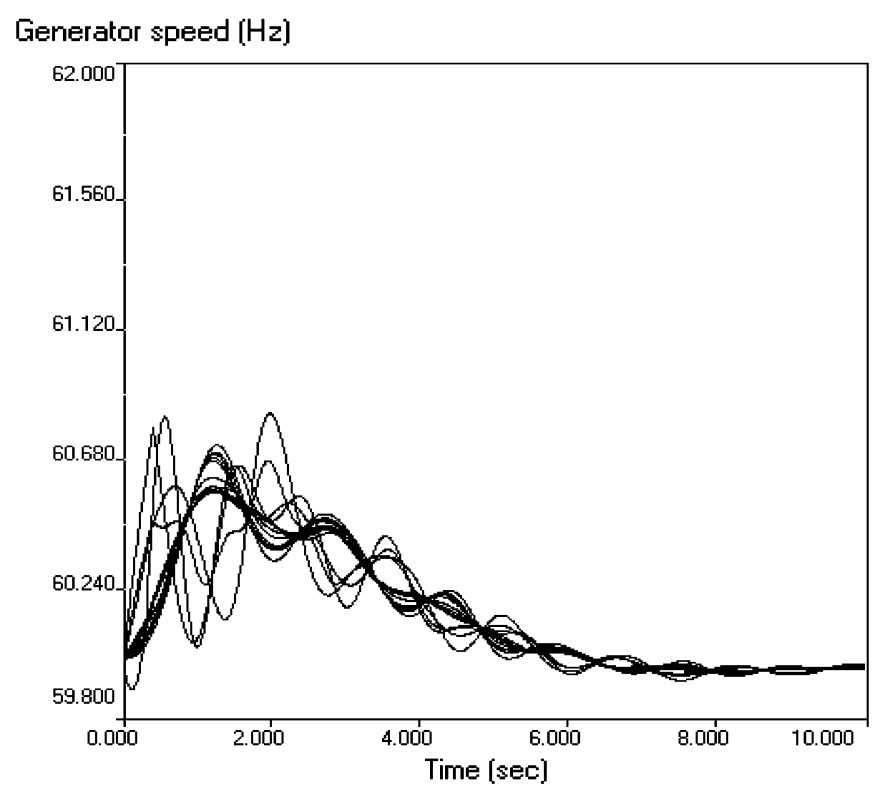

Fig. 13. System frequency for Case 2 after generator shedding.

(including the shed generator) load losses in the system. If only the quantity of load losses is considered, the splitting scheme is more efficient than the generator shedding. However, if the deviation of system frequency is also concerned, the situation will be different. Compared with the 1-Hz maximum frequency deviation for the generator shedding scheme, the splitting scheme will bring nearly $5-\mathrm{Hz}$ frequency deviation on one island. In this case, the final choice will be made based on the user-defined criterion, or other control measures, such as fast valving, can be applied first to decrease the frequency deviation.

\section{AdDitional CASES}

In this study, nearly 300 different faults were studied. A summary of results is given in Table I. All the faults presented in

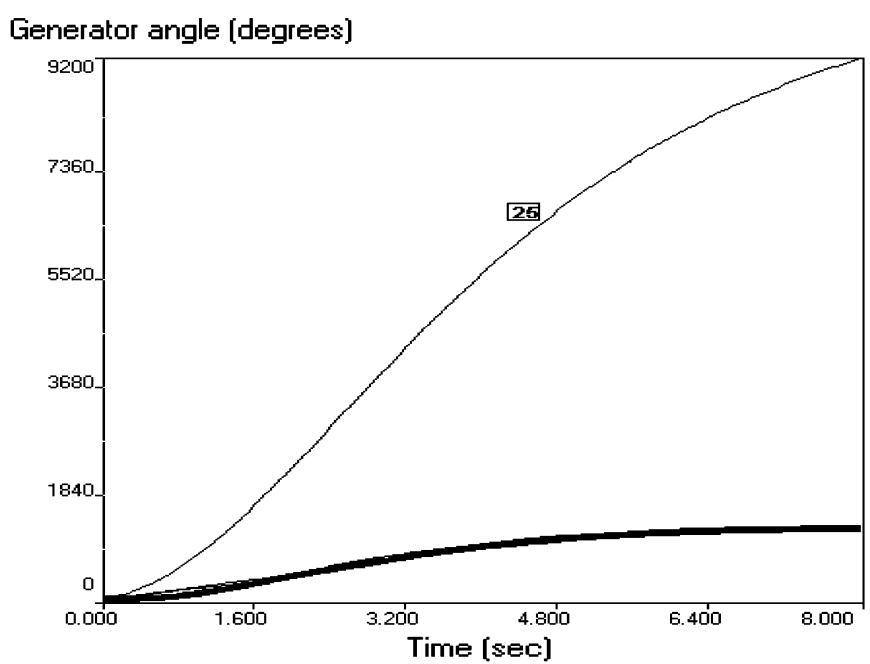

Fig. 14. Swing trajectories for Case 2 after controlling group-based splitting.

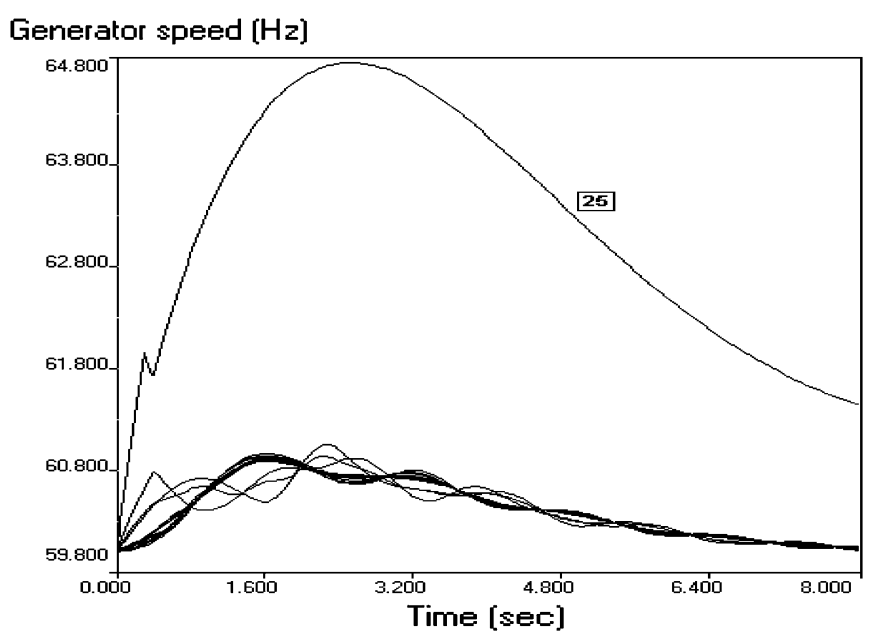

Fig. 15. System frequency for Case 2 after controlling group-based splitting.

Table I are three phase to ground and are located at the end of a transmission line (indicated by "\#” in the table).

Table I shows the simulation results after different kinds of control measures are applied to the system. All the contingencies listed in the table are the HCs with CCT less than $0.3 \mathrm{~s}$. Obviously, when the system oscillates in two-cluster mode with high coherency within each cluster, the control effects of the two splitting schemes (coherency based and controlling group based) will be exactly the same. However, when the system oscillates in multicluster mode (Cases 14, 23, and 30), the new proposed scheme is much better. The effectiveness of the unified stability control framework can also be shown by comparing the control effects between the generator shedding and system splitting schemes. Although the final decision will be made according to the user-defined criteria, in most cases, the system splitting is a competitive choice.

\section{CONCLUSIONS}

In this paper, a new system splitting (also called controlled system islanding) scheme based on the unified stability control framework is presented. The advantages and effectiveness of the new scheme have been justified through the testing cases. 
TABLE I

COMPARISON AMONG DifFERENT EMERGENCY CONTROL EFFECTS IN IEEE 118-BUS SYSTEM

\begin{tabular}{|c|c|c|c|c|c|c|c|c|c|c|}
\hline \multirow[b]{2}{*}{ No. } & \multirow[b]{2}{*}{ Faults } & \multirow{2}{*}{$\begin{array}{l}\mathrm{CCT}^{*} \\
(\mathrm{~s})\end{array}$} & \multicolumn{3}{|c|}{ New Splitting Scheme } & \multicolumn{3}{|c|}{ Coherent Splitting } & \multicolumn{2}{|c|}{ Generator Shedding } \\
\hline & & & N.I. & L.S. (MW) & A.C. & N.I. & L.S. (MW) & A.C. & N.G. & $\begin{array}{c}\text { L.G. } \\
\text { (MW) }\end{array}$ \\
\hline 1 & $89 \#-92$ & 0.0852 & 2 & 27.9 & -- & 2 & 27.9 & -- & 1 & 607 \\
\hline 2 & $26 \#-30$ & 0.1008 & 2 & 94.3 & -- & 2 & 94.3 & -- & 3 & 984 \\
\hline 3 & $88-89 \#$ & 0.1086 & 2 & 27.9 & -- & 2 & 27.9 & -- & 1 & 607 \\
\hline 4 & $8 \#-30$ & 0.1164 & 2 & 41.7 & -- & 2 & 41.7 & -- & 1 & 450 \\
\hline 5 & $85-89 \#$ & 0.1164 & 2 & 27.9 & -- & 2 & 27.9 & -- & 1 & 607 \\
\hline 6 & $89 \#-90$ & 0.1242 & 2 & 27.9 & -- & 2 & 27.9 & -- & 1 & 607 \\
\hline 7 & 89 - 92\# & 0.1633 & 2 & 27.9 & -- & 2 & 27.9 & -- & 1 & 607 \\
\hline 8 & $8-30 \#$ & 0.1711 & 2 & 41.7 & -- & 2 & 41.7 & -- & 1 & 450 \\
\hline 9 & $92 \#-100$ & 0.2102 & 2 & 27.9 & -- & 2 & 27.9 & -- & 1 & 607 \\
\hline 10 & $92 \#-102$ & 0.2102 & 2 & 27.9 & -- & 2 & 27.9 & -- & 1 & 607 \\
\hline 11 & $92 \#-93$ & 0.2102 & 2 & 27.9 & -- & 2 & 27.9 & -- & 1 & 607 \\
\hline 12 & 92\# - 94 & 0.2102 & 2 & 27.9 & -- & 2 & 27.9 & -- & 1 & 607 \\
\hline 13 & 91 - 92\# & 0.2258 & 2 & 27.9 & -- & 2 & 27.9 & -- & 1 & 607 \\
\hline 14 & $23-25 \#$ & 0.2336 & 2 & 36.0 & $\mathrm{Cl}$ & 3 & 70.0 & $\mathrm{C} 1$ & 1 & 220 \\
\hline 15 & $98-100 \#$ & 0.2336 & 2 & 6.9 & -- & 2 & 6.9 & -- & 2 & 859 \\
\hline 16 & $99-100 \#$ & 0.2336 & 2 & 6.9 & -- & 2 & 6.9 & -- & 2 & 859 \\
\hline 17 & $92-100 \#$ & 0.2414 & 2 & 6.9 & -- & 2 & 6.9 & -- & 2 & 859 \\
\hline 18 & $100 \#-103$ & 0.2414 & 2 & 6.9 & -- & 2 & 6.9 & -- & 2 & 859 \\
\hline 19 & $100 \#-104$ & 0.2414 & 2 & 6.9 & -- & 2 & 6.9 & -- & 2 & 859 \\
\hline 20 & $100 \#-106$ & 0.2414 & 2 & 6.9 & -- & 2 & 6.9 & -- & 2 & 859 \\
\hline 21 & $94-100 \#$ & 0.2414 & 2 & 6.9 & -- & 2 & 6.9 & -- & 2 & 859 \\
\hline 22 & $100 \#-101$ & 0.2414 & 2 & 6.9 & -- & 2 & 6.9 & -- & 2 & 859 \\
\hline 23 & $25 \#-27$ & 0.2492 & 2 & 43.0 & -- & 3 & 220 & $\mathrm{C} 1$ & 1 & 220 \\
\hline 24 & $49-69 \#$ & 0.2570 & 2 & 91.3 & $\mathrm{C} 1$ & 2 & 91.3 & $\mathrm{C} 1$ & 1 & 517.5 \\
\hline 25 & 69\# - 77 & 0.2570 & 2 & 91.3 & $\mathrm{C} 1$ & 2 & 91.3 & $\mathrm{C} 1$ & 1 & 517.5 \\
\hline 26 & $7-12 \#$ & 0.2570 & 2 & 41.7 & -- & 2 & 41.7 & -- & 1 & 85 \\
\hline 27 & $12 \#-14$ & 0.2570 & 2 & 41.7 & -- & 2 & 41.7 & -- & 1 & 85 \\
\hline 28 & $12 \#-16$ & 0.2570 & 2 & 41.7 & -- & 2 & 41.7 & -- & 1 & 85 \\
\hline 29 & $11-12 \#$ & 0.2570 & 2 & 41.7 & -- & 2 & 41.7 & -- & 1 & 85 \\
\hline 30 & $26-30 \#$ & 0.2570 & 2 & 63.0 & -- & 3 & 104.7 & -- & 3 & 984 \\
\hline 31 & $47-69 \#$ & 0.2570 & 2 & 134.3 & -- & 2 & 134.3 & -- & 1 & 517.5 \\
\hline 32 & $69 \#-75$ & 0.2648 & 2 & 91.3 & $\mathrm{C} 1$ & 2 & 91.3 & $\mathrm{C} 1$ & 1 & 517.5 \\
\hline 33 & $3-12 \#$ & 0.2648 & 2 & 41.7 & -- & 2 & 41.7 & -- & 1 & 85 \\
\hline 34 & $2-12 \#$ & 0.2648 & 2 & 41.7 & -- & 2 & 41.7 & -- & 1 & 85 \\
\hline 35 & $69 \#-70$ & 0.2648 & 2 & 91.3 & $\mathrm{C} 1$ & 2 & 91.3 & $\mathrm{C} 1$ & 1 & 517.5 \\
\hline 36 & $12 \#-117$ & 0.2648 & 2 & 41.7 & -- & 2 & 41.7 & -- & 1 & 85 \\
\hline 37 & $30 \#-38$ & 0.2727 & 2 & 63 & -- & 2 & 63 & -- & 1 & 450 \\
\hline 38 & $3-5 \#$ & 0.2805 & 2 & 41.7 & -- & 2 & 41.7 & -- & 1 & 450 \\
\hline 39 & $5 \#-6$ & 0.2805 & 2 & 41.7 & -- & 2 & 41.7 & -- & 1 & 450 \\
\hline 40 & $5 \#-11$ & 0.2805 & 2 & 41.7 & -- & 2 & 41.7 & -- & 1 & 450 \\
\hline 41 & $4-5 \#$ & 0.2805 & 2 & 41.7 & -- & 2 & 41.7 & -- & 1 & 450 \\
\hline
\end{tabular}

CCT: Critical Clearing Time; N.I.: Number of Islands; L.S.: Load Shedding; N.G.: Number of shed Generators; L.G.: Loss of Generation A.C.: Additional Control; $\mathrm{CH}$ : number of shed generators when additional control is necessary

* The CCT values in Table I are from the HC filter.

Under the unified stability control framework, different control measures can be assessed and compared with each other. The system splitting strategy will be applied to the system only when it is necessary or appropriate. The testing cases show that an appropriate system splitting scheme may diminish the load losses quantity significantly and may bring relatively larger frequency deviation. Compared with the conventional coherent splitting scheme, the new scheme is more effective under complex oscillation scenarios.

\section{REFERENCES}

[1] M. Pavella, D. Ernst, and D. Ruiz-Vega, Transient Stability of Power Systems: A Unified Approach to Assessment and Control. Norwell, MA: Kluwer, 2000.

[2] D. Ruiz-Vega and M. Pavella, "A comprehensive approach to transient stability control. I. Near optimal preventive control," IEEE Trans. Power Syst., vol. 18, no. 4, pp. 1446-1453, Nov. 2003.
[3] _ - "A comprehensive approach to transient stability control. II. Open loop emergency control," IEEE Trans. Power Syst., vol. 18, no. 4, pp. 1454-1460, Nov. 2003.

[4] Y. Xue, Quantitative Study of General Motion Stability and an Example on Power System Stability. Jiangsu, China: Jiangsu Sci. Technol., 1999.

[5] Y. Xue, T. Van Custem, and M. Ribbens-Pavella, "A simple direct method for fast transient stability assessment of large power systems," IEEE Trans. Power Syst., vol. 3, no. 2, pp. 400-412, May 1988.

[6] M. Jin, T. S. Sidhu, and K. Sun, "A new system splitting scheme based on the identification of controlling group," in Proc. Int. Conf. Future Power Systems, The Netherlands, Nov. 2005.

[7] K. Sun, T. S. Sidhu, and M. Jin, "Online pre-analysis and real-time matching for controlled splitting of large-scale power networks," in Proc. Int. Conf. Future Power Systems, The Netherlands, Nov. 2005.

[8] K. Sun, D.-Z. Zheng, and Q. Lu, "Splitting strategies for islanding operation of large-scale power systems using OBDD-based methods," IEEE Trans. Power Syst., vol. 18, no. 2, pp. 912-923, May 2003.

[9] _ - "A simulation study of OBDD-based proper splitting strategies for power systems under consideration of transient stability," IEEE Trans. Power Syst., vol. 20, no. 1, pp. 389-399, Feb. 2005. 
[10] S. B. Yusof, G. J. Rogers, and R. T. H. Alden, "Slow coherency based network partitioning including load buses," IEEE Trans. Power Syst., vol. 8, no. 3, pp. 1375-1382, Aug. 1993.

[11] “User Manual for TSAT V5,” Powertech Labs, Inc., Apr. 2005.

Ming Jin received the B.E. and M.Sc. degrees from the Shanghai Jiaotong University, Shanghai, China, in 1987 and 1990, respectively. Currently, he is pursuing the Ph.D. degree in the Department of Electrical and Computer Engineering at the University of Western Ontario, London, ON, Canada.

His areas of research interest include power system protection, monitoring, and control.

Tarlochan S. Sidhu (SM'94-F'04) received the B.E. (Hons.) degree from the Punjabi University, Patiala, India, in 1979 and the M.Sc. and Ph.D. degrees from the University of Saskatchewan, Saskatoon, SK, Canada, in 1985 and 1989, respectively.

Currently, he is Chair of the Department of Electrical and Computer Engineering and Professor and Hydro One Chair in Power Systems Engineering at the University of Western Ontario, London, ON, Canada. From July 1990 to June 2002, he was a Professor and Graduate Chair of the Department of Electrical Engineering at the University of Saskatchewan. He worked also with the Regional Computer Center, Chandigarh, India; Punjab State Electricity Board, India; and Bell-Northern Research Ltd., Ottawa, ON. His areas of research interest include power system protection, monitoring, and control.

Dr. Sidhu is a Fellow of the Institution of Electrical Engineers of the U.K. Fellow of the Institution of Engineers, India, and a Chartered Engineer in the U.K

Kai Sun received the B.S. degree in automation, the M.S. degree in control theory and control engineering, and the Ph.D. degree in control science and engineering from the Department of Automation at Tsinghua University, Beijing, China, in 1999, 2002, and 2004, respectively.

Currently, he is a Postdoctoral Fellow in the Department of Electrical and Computer Engineering at the University of Western Ontario, London, ON, Canada. His research interests include power systems, discrete event dynamic systems, hybrid systems, and complex large systems. 